\title{
PLATAFORMAS DIGITAIS PARA PARTICIPAÇÃO CÍVICA: INCLUSÃO DIGITAL E INOVAÇÃO SOCIAL DIGITAL
}

\author{
Clorisval Pereira Junior \\ Pontifícia Universidade Católica do Rio de Janeiro (PUC-Rio) \\ cjnior@gmail.com \\ Rejane Spitz \\ Pontifícia Universidade Católica do Rio de Janeiro (PUC-Rio) \\ rejane@puc-rio.br
}

Resumo: Neste estudo examinamos o avanço do uso de tecnologias digitais em iniciativas de inovação social no Brasil e verificamos como iniciativas de baixo para cima, bottom-up, que contam com a participação de comunidades de base da sociedade civil, estão utilizando as tecnologias digitais para tratar de problemas sociais geralmente não atendidos por empresas ou agências do governo. Observamos iniciativas que estão fazendo o uso de tecnologias emergentes, como dados abertos, hardware aberto, redes abertas e conhecimento aberto, para promover a participação cívica e a inclusão social. Para tanto, traçamos um panorama do avanço das tecnologias digitais no Brasil, com ênfase aos eventos formadores de uma cultura digital inovadora no país, e discutimos aspectos que estão influenciando uma maior abertura e democratização no uso de tecnologias digitais para a produção de soluções sociais. Ao analisar essas iniciativas bottom-up sob o prisma da inovação social digital, discutimos como o pensamento de design aberto pode beneficiar o desenvolvimento de plataformas de participação e de engajamento cívico que promovem uma sociedade mais aberta e inclusiva.

Palavras-chave: inclusão digital, inovação social digital, participação cívica, design aberto

\begin{abstract}
In this study we examine the use of digital technologies in social innovation initiatives in Brazil and we notice how bottom-up ou grassroots initiatives, which include the participation of the civil society, are using digital technologies to tackle social problems generally not addressed by companies or government agencies. We observe initiatives that are making use of emerging technologies such as open data, open hardware, open networks and open knowledge, to promote civic participation and social inclusion. We draw a panorama of the advancement of digital technologies in Brazil, focusing on events that enabled the growth of an innovative digital culture in the country, and discuss issues that are influencing the democratization of digital technologies for social production. By analyzing these bottom-up initiatives from the perspective of digital social innovation,
\end{abstract}


we discuss how we can benefit from an open design thinking in the development of digital platforms that promote participation and civic engagement towards a more open and inclusive society.

Keywords: digital inclusion, digital social innovation, civic participation, open design

\section{INTRODUÇÃO}

Em abril de 2015, o Ministério da Cultura do Brasil (MinC) utilizou sua fanpage no Facebook para divulgar o lançamento do portal Brasiliana Fotográfica, um repositório digital destinado à preservação de registros fotográficos históricos sobre o Brasil. A divulgação utilizou uma imagem rara de um casal de índios Botocudos feita por Walter Garbe em 1909. A índia na imagem exibia o torso nu e, portanto, a imagem foi censurada pelo Facebook.

Apesar da solicitação de desbloqueio da imagem feita pelo MinC, o Facebook manteve a decisão de censurá-la alegando possuir regras de conduta próprias, aplicadas globalmente sem se submeter a legislações locais. Devido ao fato, o MinC decidiu processar o Facebook tomando providências legais contra a prática de censura. Entendeu que ao aplicar termos de uso abusivos e sem transparência, o Facebook agiu de forma ilegal e arbitrária. Também entendeu que a postura da empresa feriu a Constituição da República; o Marco Civil da Internet; o Estatuto do Índio; a Convenção da Unesco sobre Proteção e Promoção da Diversidade e das Expressões culturais; além de desrespeitar a cultura, a história e a dignidade do povo brasileiro. (Sibahi, 2015). A polêmica se espalhou pela rede, o Facebook voltou atrás e desbloqueou a imagem.

Algumas semanas depois, o Ministro da Cultura publicou um artigo comentando o episódio e ressaltando a necessidade de se repensar a cultura digital. No artigo, Ferreira aponta que devemos pensar "qual tipo de sociedade gostaríamos de ver realizada a partir da onipresença das tecnologias de informação e comunicação em nosso cotidiano". (Ferreira, 2015)

Esse episódio expôs duas abordagens opostas e conflitantes sobre a nossa relação com as tecnologias de informação e comunicação cada vez mais ubíquas e pervasivas em nossa sociedade. Por um lado, o entendimento que a Internet deve ser um espaço democrático, que promove a diversidade e as expressões culturais a favor de uma sociedade mais inclusiva. E por outro, o entendimento que serviços de mídia social como Facebook são redes privadas, globais, transnacionais, com interesses comerciais e que aplicam seus próprios termos e condições, nem sempre transparentes e nem sempre em consonância com interesses públicos locais.

Neste estudo examinamos iniciativas de inovação social no Brasil, iniciativas de baixo para cima, bottom-up, que contam com a participação de comunidades de base da sociedade civil, e utilizam tecnologias digitais para tratar de problemas sociais geralmente não atendidos por empresas ou agências do governo. Estas iniciativas se aproveitam de tecnologias emergentes tais como dados abertos, hardware aberto, redes abertas e conhecimento aberto para promover participação cívica e inclusão social. Para tanto, traçamos um panorama do avanço das tecnologias digitais no Brasil com ênfase para episódios que estimularam o desenvolvimento de uma cultura digital inovadora no país e discutimos aspectos que têm influenciado uma maior abertura e 
democratização da produção de soluções que utilizam tecnologias digitais. Ao analisar as iniciativas bottom-up sob o prisma da inovação social digital, discutimos como o pensamento de design aberto pode beneficiar o desenvolvimento de plataformas de participação e do engajamento cívico que promovem uma sociedade mais aberta e inclusiva.

\section{DESENVOLVIMENTO}

\subsection{A sociedade da informação no brasil e a universalização do acesso}

A posição do Ministério da Cultura nesse episódio fez jus aos ideais de democracia, cidadania e participação social ensaiados desde o princípio da Internet no Brasil.

A rede Alternex, embriã da Internet no país foi uma iniciativa de organizações da sociedade civil, com foco em desenvolvimento social, colaboração e compartilhamento de informações. (Aguiar, 2008)

Antes de se iniciarem os serviços comerciais de Internet no país a Rede Nacional de Pesquisa (RNP) já havia implantando um backbone de rede TCP/IP conectando diversas instituições acadêmicas interessadas em troca e construção de conhecimento. (Carvalho, 2006)

No ano de 2000, o programa Sociedade da Informação no Brasil, lançado pelo Ministério da Ciência e Tecnologia propôs um plano estratégico para o desenvolvimento e a utilização dos serviços computação, comunicação e informação e de suas aplicações no país. O programa trouxe como pressuposto a importância das tecnologias de redes para geração de conhecimento e superação de desigualdades. Mais ainda, destacou a importância das ações de Inclusão Social, Alfabetização Digital e Universalização do Acesso, bem como das ações de produção de Conteúdos Digitais e Identidade Cultural. (Takahashi, 2000)

Apesar da visão positiva e otimista do potencial das tecnologias de redes discutido por autores como Castells (2000) e Lévy (1998), autores como Quéau (1998), Lanier (2013), apontam a necessidade de uma visão crítica para que essa nova tecnologia não se transforme em um novo espaço de dominação e manutenção de poder de grandes corporações e organizações transnacionais.

\subsection{Cultura digital}

O relatório Promises and Challenges of E-Democracy: Challenges of Online Citizen Engagement, publicado em 2003 pela Organisation for Economic Co-Operation and Development (OECD), analisou experiências de engajamento online em diferentes países e reconheceu a importância das TICs na mobilização para participação política e social cidadã. A principal conclusão foi que: a tecnologia é facilitadora, mas não é a solução. $O$ acesso à informação é pré-condição essencial para o engajamento, mas quantidade não significa qualidade. As principais barreiras para o engajamento cidadão online não são tecnológicas mas são culturais, organizacionais e constitucionais.

Essa também foi a percepção do Ministério da Cultura (MinC) no Brasil quando passou a defender que as tecnologias de redes precisavam ser compreendidas do ponto de vista cultural (Ferreira, 2015). O programa Pontos de Cultura, realizado pelo MinC, estimulou o uso da Internet pelos grupos componentes da "ancestral teia viva 
da cultura brasileira" que receberam kits multimídia com computadores, equipamentos de áudio visual e software livre para produzir e distribuir em rede conteúdos baseados em suas práticas e identidades culturais.

Os Pontos de Cultura trouxeram a diversidade cultural brasileira para o cenário da Internet. Grupos ligados à preservação do patrimônio imaterial (Bumba meu Boi, Coco de Umbigada, Jongo), bem como grupos ligados a expressões contemporâneas urbanas (Hip Hop, Street Art) passaram a dar uma identidade ao movimento de Cultura Digital no Brasil, trazendo consigo a ideologia do software livre, do conhecimento aberto, da colaboração, do aprendizado com parceiros (P2P: Peer to Peer), e do façavocê-mesmo (DIY: Do it Yourself).

Enquanto as primeiras iniciativas de disseminação das tecnologias de redes desenvolvidas pelo Ministério da Ciência e Tecnologia enfatizaram a infraestrutura e trataram da Inclusão Digital sob o ponto de vista da Universalização do Acesso, as iniciativas de Cultura Digital abriram uma nova fase, menos compensatória e mais exploratória. A partir da convivência com movimentos sociais que já traziam décadas de experiência com participação popular e combate às desigualdades, as iniciativas de Inclusão Digital ganharam uma nova identidade e tomaram um viés político e cultural. Práticas sociais inovadoras e particulares da cultura brasileira, tais como o mutirão e gambiarra, foram transpostas para o ambiente digital evocando a apropriação antropofágica característica da nossa cultura (Fonseca, 2014).

\subsection{Inovação social digital}

Podemos entender estas iniciativas de cultura digital, que se apropriam das tecnologias digitais e recriam seu cotidiano em prol do seu próprio desenvolvimento social, como processos de Inovação Social Digital.

Por Inovação Social, entendemos ideias, estratégias, produtos, serviços, modelos e organizações que simultaneamente atendem à necessidade social pelo bem comum, como também criam novas relações e colaborações fortalecendo a própria capacidade de ação social. (Mulgan, 2010).

Essas iniciativas de Inovação Social estariam tomando proporções sem precedentes, tanto por conta da maior quantidade e complexidade de problemas sociais causados pelo aumento populacional, bem como por conta das tecnologias digitais que proporcionam novas formas de conexão e de colaboração (Manzini, 2015).

Neste sentido, entendemos a Inovação Social Digital, como ações sociais em prol do bem comum, que envolvem participação cívica, mobilização coletiva, colaboração, compartilhamento de recursos e descentralização de poder, e que são empoderadas por tecnologias digitais emergentes baseadas em plataformas abertas, seguindo tendências tecnológicas inovadoras como Open Data, Open Hardware, Open Networks e Open Knowledge. (Bria et al., 2014)

O crescimento da Internet se deu em um cenário de emergência criativa e inovação de baixo para cima, baseado em princípios de neutralidade da rede, arquitetura P2P, infraestrutura aberta e distribuída. Ecossistemas digitais abertos e distribuídos se mostram mais apropriados para promover e encorajar inovações alinhadas com as necessidades sociais de cidadãos e de comunidades.

No entanto, o volume de dados produzidos e processados por plataformas abertas e destinados à inovação social ainda é pequeno quando comparado às iniciativas de grandes corporações que atuam no mercado globalizado e que tratam os 
dados de forma proprietária. Apesar da infraestrutura de rede disponível para acesso e inclusão de inciativas 'bottom-up', os serviços comerciais implantados nesta rede continuam a privilegiar iniciativas 'top-down', de grandes organizações centralizadas e estabelecidas no governo e no setor corporativo (Bria et al., 2014).

Pesquisas recentes apontam uma tendência global e crescente de aumento de investimentos em Big Data (Gartner, 2014). A Associação Brasileira de Empresas de Software reportou investimentos em Big Data da ordem de $\mathrm{R} \$ 853$ milhões para o ano 2014 no Brasil (ABES, 2014).

Por outro lado, podemos notar uma emergência de organizações que estão vislumbrando um outro futuro possível a partir da promoção, estimulo, apoio e aceleração de iniciativas de Inovação Social Digital que melhor atendem às necessidades de cidadania e coletividade

Essas iniciativas de Inovação Social Digital estão reinventando a forma como nossa sociedade lida com os problemas de superpopulação e escassez de recursos no planeta. Estão mudando o foco para o que temos em abundância: as pessoas. Em vez de focar na carência, passam a trabalhar com a potência das comunidades e das conexões sociais. Estão transformando informação e conhecimento em um bem comum.

\subsection{Design para todos e com todos}

Este cenário de Inovação Social Digital reforça a discussão crítica sobre o papel social do design o que tem sido debatida desde a segunda metade do século XX por autores como: Papanek (1971); Deforges (1994); Findeli (2001); Heller e Vienne (2003); Manzini e Jegou (2004); Thackara (2005). Esses autores discutem propostas e visões alternativas ao paradigma materialista da produção industrial e urgem pela responsabilidade social na prática do design.

A discussão sobre Design orientado para competitividade industrial em contraposição ao Design orientado ao desenvolvimento social expõe a oposição entre duas correntes teóricas contemporâneas no campo do design caracterizadas pelo pensamento de Herbert Simon e de Richard Buchanan (DiSalvo, 2009). Simon aproxima o processo de design à ciência da tomada de decisões, colocando o design em um patamar racionalista muito útil à busca pela eficiência na produção industrial. Buchanan caracteriza o design como uma forma contemporânea de retórica preocupada em comunicar crenças e incitar a ação através de argumentos sobre como devemos dirigir nossa vida, abrindo a prática do design à crítica ética, moral e política (DiSalvo, 2012).

Para Manzini (2015) a abordagem racional defendida por Simon, estaria alinhada um modo de projeto focado em soluções de problemas (Problem Solving) para o nosso mundo físico. Já a abordagem retórica defendida por Buchanan estaria alinhada a um modo de projeto focado em significação e construção de sentidos (Sense Making) mais adequado ao design para o social. Mais do que polarizar essas abordagens, Manzini observa que no cenário de Inovação Social, essas diferentes abordagens tendem a convergir em especial pelo fato de que cada vez mais o desenvolvimento do projeto de design passa a ser uma atividade aberta e compartilhada. A solução de problemas em projetos sociais depende de uma construção de sentidos coletiva. 
Dessa forma podemos vislumbrar cenários em que pessoas, cidadãos e comunidades deixam de ser audiência, deixam de ser meros consumidores, e se tornam produtores da sua própria realidade utilizando ferramentas abertas e distribuídas que promovem a colaboração, a construção de narrativas e a construção da inteligência coletiva e compartilhada (Levy, 2007).

\subsection{Iniciativas de Inovação Social Digital no Brasil}

Novos cenários possíveis estão surgindo a partir dessas experiências de Inovação Social Digital, que estão promovendo Inclusão Digital, Cultura Digital, com foco no desenvolvimento de plataformas de participação cívica que se apoiam em tecnologias emergentes como: Open Data; Open Hardware; Open Networks; e Open Knowledge.

Essas soluções permitem maior a colaboração entre cidadãos e administração pública, incrementando a comunicação entre ambas as partes, de forma qualitativa e quantitativa, bem como promovendo maior satisfação com o uso dos serviços públicos (Rosa, 2014).

A partir de um estudo sobre iniciativas de Inovação Social Digital na Comunidade Europeia e América do Norte, Bria et al. (2014) propuseram um framework dividindo as iniciativas Inovação Social em 6 domínios: Democracia Eletrônica; Acesso Aberto; Economia Colaborativa; Redes de Conscientização; Novos Modos de Fabricação; Financiamento, Incubação e Aceleração (Bria et al. 2014). Com base nesse framework, realizamos um mapeamento de uma série iniciativas de Inovação Social Digital no Brasil conforme descrito a seguir.

\subsubsection{Democracia Eletrônica}

São iniciativas baseadas em tecnologias digitais abertas, que inovam os modelos tradicionais de democracia representativa, permitindo a participação coletiva dos cidadãos em processos de tomada de decisão, deliberação coletiva e mobilização de massa, em uma escala muito maior do que anteriormente possível com tecnologias tradicionais. Exemplos de iniciativas de democracia aberta incluem: Plataformas de Transparência; Legislação Participativa; Deliberação e Tomada de Decisão Participativa; Plataformas de Petição; Ocorrências e Atendimento ao Cidadão; Sistemas de Votação Online.

\begin{tabular}{l|l}
\hline Iniciativas Globais (Bria et al., 2014) & Iniciativas no Brasil \\
\hline Open Spending & Plataforma Brasil \\
https://openspending.org & http://plataformabrasil.org \\
Finnish Open Ministry & Legislando \\
http://openministry.info & http://legislando.nossascidades.org \\
Avaaz & Panela de Pressão \\
http://www.avaaz.org & http://paneladepressao.nossascidades.org \\
SeeClickFix & PortoAlegre.cc \\
http://seeclickfix.com & http://portoalegre.cc \\
FixMyStreet & Colab.re \\
http://www.colab.re & http://www.colab.re \\
\hline
\end{tabular}

Tabela 01: Iniciativas de Democracia Eletrônica (E-Democracy) 


\subsubsection{Acesso Aberto}

São iniciativas que oferecem acesso livre a conteúdo, com padrões abertos e licenciamento aberto. São iniciativas no campo do conhecimento comum e dos direitos digitais, com potencial para empoderar os cidadãos e aumentar a participação cívica, ao mesmo tempo preservando as características de abertura e acessibilidade das infraestruturas de redes. Exemplos de iniciativas de Acesso Aberto incluem: Dados Abertos; Redes Distribuídas; Transparência; Redes Abertas de Colaboração e Aprendizagem.

\begin{tabular}{l|l}
\hline Iniciativas Globais (Bria et al., 2014) & Iniciativas no Brasil \\
\hline Apps for Democracy & Hackaton Rio Apps Open Data \\
https://isl.co/work/apps-for-democracy/ & http://riodatamine.com.br/ \\
Chaos Computer Club & Hackaton 1746 Data.Rio Open Data \\
https://www.ccc.de/en/ & http://data.rio/ \\
Observe Hack Make & Open Knowledge Brasil \\
https://ohm2013.org/ & http://br.okfn.org \\
City SDK & Matéria Brasil \\
http://www.citysdk.eu & http://www.materiabrasil.com \\
Github & Software Livre Brasil \\
http://github.io & http://softwarelivre.org \\
& Transparência Hacker \\
& https://www.facebook.com/transparencia.hacker \\
& Redes Livres \\
& http://www.redeslivres.org.br \\
\hline
\end{tabular}

Tabela 02: Iniciativas de Acesso Aberto (Open Access)

\subsubsection{Economia Colaborativa}

São iniciativas que utilizam tecnologias digitais para promover modelos socioeconómicos colaborativos que permitir que o compartilhamento de habilidades, conhecimento, alimentação, vestuário, habitação, etc. São plataformas de intercâmbio e compartilhamento de recursos baseadas em reputação e confiança. Exemplos de iniciativas de Economia Colaborativa sãos: Plataformas de compartilhamento de ativos e recursos; Financiamento colaborativo; Plataformas de Redução de Consumo; Moedas Alternativas; Marketplaces digitais.

\begin{tabular}{l|l}
\hline Iniciativas Globais (Bria et al., 2014) & Iniciativas no Brasil \\
\hline P2P Foundation & Benfeitoria \\
http://p2pfoundation.net/ & http://benfeitoria.com \\
Ouishare & Tem Açucar \\
http://ouishare.net & http://www.temacucar.com \\
Goteo & Bliive \\
http://goteo.org & http://bliive.com \\
Peerby & Atados \\
http://peerby.org & https://www.atados.com.br \\
& Conectando Gerações \\
& http://www.conectandogeracoes.com.br \\
& Banco de Remédios \\
& https://www.facebook.com//Banco-de- \\
& Remédios/ \\
\hline
\end{tabular}

Tabela 03: Iniciativas de Economia Colaborativa (Collaborative Economy) 


\subsubsection{Redes de Conscientização}

São uma nova geração de produtos e serviços que estão agregando dados provenientes de pessoas e do ambiente, a fim de promover empoderamento e mudança comportamental de indivíduos e comunidades. Exemplos de iniciativas de Redes de Conscientização incluem: Redes Abertas de Sensores; Mapeamentos de Crises; Mapeamentos Voluntários; Redes de Atenção pública; Planejamento Urbano Participativo; Comunidades de Cuidado.

\begin{tabular}{l|l}
\hline Iniciativas Globais (Bria et al., 2014) & Iniciativas no Brasil \\
\hline Safecast & WikiMapa \\
http://safecast.org & http://wikimapa.org.br \\
Smart Citizen Kit & Tá no Mapa \\
https://smartcitizen.me & http://www.tanomapa.org \\
Everyaware & Rede InfoAmazonia \\
http://www.everyaware.eu & http://infoamazonia.org \\
Ushahidi & Cidade Azul \\
http://ushahidi.org & http://www.cidadeazul.org \\
Wikiprogress & De Guarda \\
http://www.wikiprogress.org & http://deguarda.nossascidades.org \\
Patients Like Me & Onde fui roubado \\
https://www.patientslikeme.com & http://www.ondefuiroubado.com.br \\
\hline
\end{tabular}

Tabela 04: Iniciativas de Redes de Conscientização (Awareness Networks)

\subsubsection{Novos Modos de Fabricação}

São comunidades que utilizam ferramentas de fabricação livre e código aberto, tais como Impressoras 3D, CNC, CAD/CAM, e dão acesso tanto a infraestruturas, produtos, como de aprendizagem e desenvolvimento de habilidades e capacidades necessárias para o trabalho colaborativo. São exemplos de Novos Modos de Fabricação: Fablabs; Maker Spaces; Cultura DIY; Hacking e Tinkering; Open Source; Open Design.

\begin{tabular}{l|l}
\hline Iniciativas Globais (Bria et al., 2014) & Iniciativas no Brasil \\
\hline Maker Faire & Fablab Brasil \\
http://makerfaire.com & http://www.fablabbrasil.org \\
Fab Foundation & Garagem FabLab \\
http://www.fabfoundation.org/fab-labs/ & http://www.garagemfablab.com \\
Arduino & OHMS \\
http://arduino.org & https://www.facebook.com/ourhomemakerspace \\
& Olabi \\
& http://olabi.co \\
& HackLab \\
& http://www.hacklab.com.br/ \\
\hline
\end{tabular}

Tabela 05: Iniciativas de Novos Modos do Fabricação (New Ways of Making)

\subsubsection{Financiamento, Incubação e Aceleração.}

Incubadoras, aceleradores criados pelos financiadores públicos e privados podem apoiar projetos de inovação social digital através de uma combinação de financiamentos e apoio não financeiro como acesso a espaços de co-working, apoio empresarial e mentoring. Exemplos de iniciativas de Financiamento, Incubação e 
Aceleração incluem: Financiamento Semente; Financiamento Colaborativo; Startups; Mentoring; Desafios e Prêmios.

\begin{tabular}{l|l}
\hline Iniciativas Globais (Bria et al., 2014) & Iniciativas no Brasil \\
\hline Nesta & Social Good Brazil \\
http://nesta.org.ok & http://socialgoodbrasil.org.br \\
Code For America & Rio + \\
http://www.codeforamerica.org & http://riomais.benfeitoria.com \\
Y Combinator & Awesome Foundation Rio \\
http://www.purpose.com & http://www.awesomefoundation.org/ \\
Seed Bootcamp & Purpose Brasil \\
http://www.purpose.com & http://www.purpose.com \\
\hline
\end{tabular}

Tabela 06: Iniciativas de Financiamento, Incubação e Aceleração (Funding, Incubation, Acceleration)

\section{CONSIDERAÇÕES FINAIS}

Conforme apresentado no estudo, observamos diversas iniciativas no Brasil que estão explorando tecnologias digitais e o potencial de conexão e colaboração das redes digitais, para construir ecossistemas de participação cívica baseados promovendo o conhecimento aberto e maior participação e inclusão social, assim como maior bem estar social.

A partir deste mapeamento exploratório inicial, podemos observar que a tendência de desenvolvimento de iniciativas de Inovação Social Digital observada em países da Comunidade Europeia e América do Norte (Bria et al. 2014) também pode ser observada no Brasil e na América Latina.

Estas iniciativas, estão construindo uma sociedade mais justa e democrática, e oferecendo maior oportunidade de participação, onde pessoas, cidadãos e comunidades possuem voz e estão empoderadas para colaborar em cenários de governo aberto e de planejamento urbano participativo, bem como deliberar sobre a construção da sua própria realidade.

Conforme foi possível observar neste estudo, essas iniciativas são em sua maioria lideradas pela sociedade civil, ONGs, associações comunitárias locais, grupos de pesquisa acadêmica, tecnológica e científica ou por Startups focadas no empreendedorismo social.

Um aprofundamento desse estudo irá avaliar o estágio de Inovação Social em que se encontra cada iniciativa, considerando os 6 estágios do processo de Inovação Social (Murray et al, 2010), detalhar aspectos relacionados às soluções tecnológicas adotadas, tais como forças e fraquezas, bem como analisar aspectos mais específicos de design

Este cenário de Inovação Social Digital traz novos desafios para o campo do Design. Percebemos que para colaborar nesse cenário de Inovação Social Digital é fundamental que se abrace uma nova cultura de Design onde sejam trabalhados temas como: Design Aberto; Design como Ativismo; Design Participativo e Co-Design.

Assim podemos vislumbrar novas formas de pensar e de fazer design. Deixando de lado o determinismo e da eficiência industrial e se abrindo para a ação e a transformação social. 


\section{REFERÊNCIAS}

ABES. As perspectivas e desafios do Big Data no Brasil. Associação Brasileira de Empresas de Software. Disponível em: <http://www.abessoftware.com.br/noticias/asperspectivas-e-desafios-do-big-data-no-brasil>. Acessado em: 18/12/2014.

AGUIAR, S. Redes sociais: da mobilização popular para o ativismo digital. In: Das ruas às redes. 15 anos de mobilização social na luta contra a fome e a pobreza. (Coleção Cidadania em rede). Rio de Janeiro: COEP. 2008

BRIA, F.; SESTINI, F.; GASCO, M.; BAECK, P.; HALPIN, H.; ALMIRALL, E.; KRESIN, F. Growing a Digital Social Innovation Ecosystem for Europe: DSI Final Report. European Union, 2015. Disponível em:

$<$ http://issuu.com/digitalsocialinnovation/docs/dsireport7forwebsite7print $>$. Acessado em 23/02/2015

CARVALHO, M. A trajetória da Internet no Brasil: do surgimento das redes de computadores à instituição dos mecanismos de governança. Dissertação de mestrado. COPPE/UFRJ. 2006

CASTELLS, M. A Sociedade em Rede. Editora Paz e Terra, São Paulo. 2000

DEFORGES, Y. Por um Design Ideológico. In: Estudos em Design. Volume II, Número 1. 1994

DISALVO, C. Design and the Construction of Publics. In: Design Issues: Volume 25, Number 1 Winter 2009. Massachusetts Institute of Technology. 2009

DISALVO, C. Adversarial Design. The MIT Press. Kindle Edition. 2012

FERREIRA, J. É hora de repensar a cultura digital. Brasil Post. Publicado: 07/05/2015.

Acessado: 10/05/2015. http://www.brasilpost.com.br/juca-ferreira/e-hora-derepensar-a-cultura-digital_b_7222272.html?utm_hp_ref=brazil e http://culturadigital.br/jucaferreira/2015/05/07/hora-de-repensar-a-cultura-digital/

FINDELI, A. Rethinking Design Education for the 21st Century: Theoretical, Methodological, and Ethical Discussion. In: Design Issues. Volume 17, Number 1 Winter 2001. Massachusetts Institute of Technology.

FONSECA, F. REDELABS: Laboratórios Experimentais em Rede. Master dissertation. IEL/Labjor, da Universidade Estadual de Campinas. 2014

GARTNER. Gartner Survey Reveals That 73 Percent of Organizations Have Invested or Plan to Invest in Big Data in the Next Two Years. Gartner.

<http://www.gartner.com/newsroom/id/2848718>, 17/09/2014.

HELLER, S.; VIENNE, V. Citizen Designer: Perspectives on Design Responsibility. Allworth Press. Kindle Edition. 2003

LANIER, J. Who Owns the Future? San Jose: Simon \& Schuster; UK: Allen Lane. 2013

LEVY, P. As tecnologias da inteligência: $O$ futuro do pensamento na era da informática. Editora 34, São Paulo. 1998

OECD. Promises and Challenges of E-Democracy: Challenges of Online Citizen Engagement. OECD. 2003 
MANZINI, E.; JÉGOU, F. Sustainable Everyday: Scenarios for Urban Life. Milan: Edizioni Ambiente. 2003

MANZINI, E. Design When Everybody Designs: An Introduction to Design for Social Innovation. Massachusets: MIT Press. 2015

MULGAN, G. Social Innovation: What it is, why it matters and how it can be accelerated. Skoll Centre for Social Entrepreneurship, University of Oxford. 2010 MURRAY, R.; CAULIER-GRICE, J; MULGAN, G. The Open Book of Social Innovation. Nesta, The Young Foundation. 2010 Disponível em: http://www.nesta.org.uk/sites/default/files/the_open_book_of_social_innovation.pdf . Acessado em Abril, 2016.

PAPANEK, V. Design for the Real World: Human Ecology and Social Change. New York: Pantheon Books. 1971

QUÉAU, P. The Information Revolution: in sarch of the common good. Conferência Internacional Mídia e Percepção Social, 18-20 de maio de 1998, Instituto do Pluralismo Cultural, Universidade Candido Mendes, Rio de Janeiro. 1998

ROSA, R.. Tecnologías cívicas y participación ciudadana. In: Revista de Estudios de Juventud. Periodismo Ciudadano. Nuevas formas de comunicación, organización e información. Junio 14 | no 105. Coordinador: Oscar Espiritusanto. Universidad Carlos III de Madrid. 2014

SIBAHI, P. Ministério da Cultura vai processar o Facebook por censurar fotos de indígenas. Brasil Post. Publicado: 17/04/2015. Acessado: 20/04/2015.

http://www.brasilpost.com.br/2015/04/17/ministerio-da-cultura-processafacebook_n_7089848.html

TAKAHASHI, T. Sociedade da informação no Brasil: Livro verde. Ministério da Ciência e Tecnologia, Brasil. 2000

THACKARA, J. In the bubble: designing in a complex world. Massachusetts Institute of Technology. 2005. 